\title{
ASYMPTOTIC ESTIMATES FOR EIGENVALUES OF RIGHT DEFINITE TWO PARAMETER STURM-LIOUVILLE PROBLEMS
}

\author{
by PATRICK J. BROWNE* and B. D. SLEEMAN
}

(Received 2nd August 1991)

Precise asymptotic estimates for the eigenvalues of a uniformly right definite two parameter system of Sturm-Liouville problems are developed. The work extends recent results of B. P. Rynne.

1991 Mathematics subject classification: 34B24, 34E05

\section{Introduction}

In a recent interesting paper [6], Rynne gave a new approach to the question of obtaining asymptotic estimates for the eigenvalues of a uniformly right definite multiparameter system of Sturm-Liouville problems. As he pointed out, Faierman, $[3,4]$, earlier, presented an account for two parameter systems obtaining more precise results but at the cost of restrictive hypotheses and considerable complexity of argument. Rynne's return to this important problem in multiparameter spectral theory is thus welcome and opens the way for further study. It is our intent in this note to use Rynne's main ideas on a two parameter problem (in the style of Faierman) and to sharpen Rynne's estimates at least for those eigenvalues falling in a certain region described below.

Specifically, we consider the two parameter system

$$
\begin{aligned}
& y_{1}^{\prime \prime}\left(x_{1}\right)+q_{1}\left(x_{1}\right) y_{1}\left(x_{1}\right)+\left(\lambda A_{1}\left(x_{1}\right)-\mu B_{1}\left(x_{1}\right)\right) y_{1}\left(x_{1}\right)=0, \quad 0 \leqq x_{1} \leqq 1, \\
& y_{2}^{\prime \prime}\left(x_{2}\right)+q_{2}\left(x_{2}\right) y_{2}\left(x_{2}\right)+\left(-\lambda A_{2}\left(x_{2}\right)+\mu B_{2}\left(x_{2}\right)\right) y_{2}\left(x_{2}\right)=0, \quad 0 \leqq x_{2} \leqq 1,
\end{aligned}
$$

subject to Dirichlet boundary conditions

$$
y_{1}(0)=y_{1}(1)=y_{2}(0)=y_{2}(1)=0 \text {. }
$$

The coefficient functions $q_{1}, q_{2}$ are assumed to be real valued and continuous while

*Research supported in part by a grant from the NSERC of Canada. 
$A_{1}, B_{1}, A_{2}, B_{2}$ are real valued and twice continuously differentiable. We also impose the so called "uniform right definiteness condition", viz

$$
\operatorname{det}\left[\begin{array}{rr}
A_{1}\left(x_{1}\right) & -B_{1}\left(x_{1}\right) \\
-A_{2}\left(x_{2}\right) & B_{2}\left(x_{2}\right)
\end{array}\right]>0, \text { for all } x_{1}, x_{2} \in[0,1]
$$

under which there is no loss of generality in assuming that $A_{1}, B_{1}, A_{2}, B_{2}$ are all positive functions-see [1, Theorem 2].

An eigenvalue for the system (1), (2) is a pair of (necessarily real) numbers $\left(\lambda^{*}, \mu^{*}\right)$ such that for $\lambda=\lambda^{*}, \mu=\mu^{*}$, both equations in (1) can be solved non-trivially with $y_{1}, y_{2}$ satisfying the boundary conditions (2). The principal result concerning the existence of eigenvalues for our problem is Klein's oscillation theorem quoted by Rynne [6, Theorem 1.1] which guarantees the existence of a sequence of eigenvalues $\lambda=(\lambda, \mu)$ and enumerates them as $\lambda^{i}$ where $\mathbf{i}=(i, j), i, j$ non-negative integers, so that corresponding to $\lambda^{i}$, the first (respectively, second) equation of (1) has a solution with $i$ (respectively, $j$ ) zeros in the open interval $(0,1)$. It is our aim here to give asymptotic estimates for $\lambda^{i}$ when $i$ is large (in the sense that $\|\mathbf{i}\|=\left(i^{2}+j^{2}\right)^{1 / 2}$ is large). We have chosen Dirichlet boundary conditions (2) for convenience: our results extend to any separated self-adjoint boundary conditions.

Each of us is grateful to the other's institution for hospitality extended during recent reciprocal visits.

\section{Preliminary formulation and results}

We begin by introducing the sector $\mathbf{R}^{2}$ in which our asymptotic analysis will be valid. For a general discussion on the location of the eigenvalues we cite Binding and Browne [2]. Initially, consider the cone $D \subset \mathbf{R}_{+}^{2}$ given by

$$
D=\left\{(\lambda, \mu) \in \mathbf{R}^{2} \mid \lambda A_{1}\left(x_{1}\right)-\mu B_{1}\left(x_{1}\right)>0, \forall x_{1} \in[0,1],-\lambda A_{2}\left(x_{2}\right)+\mu B_{2}\left(x_{2}\right)>0, \forall x_{2} \in[0,1]\right\} .
$$

Note that for $(\lambda, \mu) \in D$ we have

$$
t_{0}:=\sup _{x_{2}}\left(\frac{A_{2}\left(x_{2}\right)}{B_{2}\left(x_{2}\right)}\right)<\frac{\mu}{\lambda}<\inf _{x_{1}}\left(\frac{A_{1}\left(x_{1}\right)}{B_{1}\left(x_{1}\right)}\right):=t_{1} .
$$

The uniform right definiteness condition (3) ensures that $t_{0}<t_{1}$. We select a suitably small $\delta>0$ and define $D_{\delta}$ by

$$
D_{\delta}=\left\{(\lambda, \mu) \in D \mid \mu / \lambda=t \in\left[t_{0}+\delta, t_{1}-\delta\right]\right\} .
$$

For $(\lambda, \mu) \in D$ such that $t \in\left(t_{0}, t_{1}\right)$ we also see that the functions

$$
s_{1}\left(x_{1}, t\right)=A_{1}\left(x_{1}\right)-t B_{1}\left(x_{1}\right), \quad s_{2}\left(x_{2}, t\right)=-A_{2}\left(x_{2}\right)+t B_{2}\left(x_{2}\right)
$$


are both positive. This enables us to use the Liouville transformation on the equations (1). For a given $t \in\left(t_{0}, t_{1}\right)$ and $i=1,2$, we define

$$
\begin{gathered}
k_{i}=\int_{0}^{1} s_{i}\left(x_{i}, t\right)^{1 / 2} d x_{i}, \\
z_{i}=k_{i}^{-1} \int_{0}^{x_{i}} s_{i}(\eta, t)^{1 / 2} d \eta, \\
u_{i}=s_{i}^{1 / 4} y_{i},
\end{gathered}
$$

to obtain the pair of equations

$$
\begin{gathered}
-\ddot{u}_{i}+\left\{-k_{i}^{2} q_{i} s_{i}^{-1}+k_{i}^{2} s_{i}^{-1 / 4}\left(s_{i}^{1 / 4}\right)^{\cdot *}\right\} u_{i}=\lambda k_{i}^{2} u_{i}, \\
u_{i}(0)=u_{i}(1)=0, \quad i=1,2 .
\end{gathered}
$$

Here

$$
\cdot=\frac{d}{d z_{i}}
$$

and in what follows,

$$
\left[f_{i}\right]=\int_{0}^{1} f\left(z_{i}\right) d z_{i}
$$

The well known characterization of Dirichlet eigenvalues (see e.g. [5, Theorem 9, p. 35]) can be invoked so that for $i=1$, the problem (4) has a sequence of eigenvalues $\lambda_{m}^{1}(t)$ satisfying

$$
k_{1}^{2} \lambda_{m}^{1}(t)=m^{2} \pi^{2}-k_{1}^{2}\left[q_{1} / s_{1}\right]+k_{1}^{2}\left[\frac{\left(s_{1}^{1 / 4}\right)^{\cdot \cdot}}{s_{1}^{1 / 4}}\right]+a_{m}^{1}(t), \quad m \geqq 1,
$$

where $\sum_{m}\left|a_{m}^{1}(t)\right|^{2}<\infty$, while for $i=2,(4)$ has eigenvalues $\lambda_{n}^{2}(t)$ satisfying

$$
k_{2}^{2} \lambda_{n}^{2}(t)=n^{2} \pi^{2}-k_{2}^{2}\left[q_{2} / s_{2}\right]+k_{2}^{2}\left[\frac{\left(s_{2}^{1 / 4}\right)^{\cdots}}{s_{2}^{1 / 4}}\right]+a_{n}^{2}(t), \quad n \geqq 1,
$$

where $\sum_{n}\left|a_{n}^{2}(t)\right|^{2}<\infty$.

We shall need the mapping $\phi: \mathbf{R}^{2} \rightarrow \mathbf{R}_{+}^{2}$ used by Rynne:

$$
\phi_{1}(\lambda, \mu)=\int_{0}^{1}\left(\lambda A_{1}\left(x_{1}\right)-\mu B_{1}\left(x_{1}\right)\right)_{+}^{1 / 2} d x_{1}
$$




$$
\phi_{2}(\lambda, \mu)=\int_{0}^{1}\left(-\lambda A_{2}\left(x_{2}\right)+\mu B_{2}\left(x_{2}\right)\right)_{+}^{1 / 2} d x_{2}
$$

Here $f_{+}$denotes the positive part of $f$, but when $(\lambda, \mu) \in D$ this becomes irrelevant. Important properties of the mapping $\phi$ are given by Rynne in [6, Lemmas 2.5, 2.6, Theorem 3.1]. The set $Q$ is defined as

$$
Q=\left\{(\lambda, \mu) \mid \phi_{i}(\lambda, \mu)>0, i=1,2\right\}
$$

Of course we have $D_{\delta} \subset D \subset Q$.

The following observations will be useful in the sequel. With reference to equations (5) and (6) we have

$$
\begin{aligned}
{\left[q_{1} / s_{1}\right] } & =\int_{0}^{1} \frac{q_{1}}{s_{1}} d z_{1}=k_{1}^{-1} \int_{0}^{1} \frac{q_{1}}{s_{1}} s_{1}^{1 / 2} d x_{1} \\
& =\left(\int_{0}^{1}\left(A_{1}\left(x_{1}\right)-t B_{1}\left(x_{1}\right)\right)^{1 / 2} d x_{1}\right)^{-1} \int_{0}^{1} \frac{q_{1}\left(x_{1}\right) d x_{1}}{\left(A_{1}\left(x_{1}\right)-t B_{1}\left(x_{1}\right)\right)^{1 / 2}} \\
& =\frac{\lambda^{1 / 2}}{\phi_{1}(\lambda, \mu)} \int_{0}^{1} \frac{q_{1}\left(x_{1}\right) d x_{1}}{\left(A_{1}\left(x_{1}\right)-t B_{1}\left(x_{1}\right)\right)^{1 / 2}} .
\end{aligned}
$$

and similarly,

$$
\left[q_{2} / s_{2}\right]=\frac{\lambda^{1 / 2}}{\phi_{2}(\lambda, \mu)} \int_{0}^{1} \frac{q_{2}\left(x_{2}\right) d x_{2}}{\left(-A_{2}\left(x_{2}\right)+t B_{2}\left(x_{2}\right)\right)^{1 / 2}}
$$

Here, as before, we have used $\mu / \lambda=t$. Parallel calculations show

$$
\begin{gathered}
{\left[\frac{\left(s_{1}^{1 / 4}\right)^{\cdot \cdot}}{s_{1}^{1 / 4}}\right]=\frac{\phi_{1}(\lambda, \mu)}{\lambda^{1 / 2}} \int_{0}^{1}\left[\frac{A_{1}^{\prime \prime}-t B_{1}^{\prime \prime}}{4\left(A_{1}-t B_{1}\right)^{3 / 2}}-\frac{5}{16} \frac{\left(A_{1}^{\prime}-t B_{1}^{\prime}\right)^{2}}{\left(A_{1}-t B_{1}\right)^{5 / 2}}\right] d x_{1} .} \\
{\left[\frac{\left(s_{2}^{1 / 4}\right)^{\cdot \cdot}}{s_{2}^{1 / 4}}\right]=\frac{\phi_{2}(\lambda, \mu)}{\lambda^{1 / 2}} \int_{0}^{1}\left[\frac{-A_{2}^{\prime \prime}+t B_{2}^{\prime \prime}}{4\left(-A_{2}+t B_{2}\right)^{3 / 2}}-\frac{5}{16} \frac{\left(-A_{2}^{\prime}+t B_{2}^{\prime}\right)^{2}}{\left(-A_{2}+t B_{2}\right)^{5 / 2}}\right] d x_{2} .}
\end{gathered}
$$

We close this section with two hypotheses used by Rynne which we too shall need.

Hypothesis F. Suppose that $f$ is a real valued $C^{2}$ function defined on $U=[0,1]$. We say that $f$ satisfies hypothesis $F$ if the set $\{x \in U \mid f(x)>0\}$ can be decomposed into the 
union of a finite number of disjoint open intervals $I_{i}=\left(a_{i}^{1}, a_{i}^{2}\right), i=1, \ldots, n$, (together with any of the end points $a_{i}^{j}$ which are not zeros of $f$ ) and there exists a constant $K>1$ such that on each interval $I_{i}$, either

(i) $\frac{\left|\left(x-a_{i}^{1}\right) f^{\prime}(x)\right|}{f(x)} \leqq K, \quad \frac{\left|\left(x-a_{i}^{1}\right)^{2} f^{\prime \prime}(x)\right|}{f(x)} \leqq K$,

(ii) there is an increasing function $\widetilde{f}_{i}$ such that

$$
K^{-1} \tilde{f}_{i}(x) \leqq f(x) \leqq K f_{i}(x),
$$

or (i) holds with $a_{i}^{1}$ replaced by $a_{i}^{2}$ and (ii) holds with $f_{i}$ decreasing.

Hypothesis UF. Suppose that the function $f: U \times X \rightarrow \mathbf{R}$ is such that for each $\xi \in X$, the function $f(\cdot, \xi): U \rightarrow \mathbf{R}$ satisfies hypothesis $F$ and let $n(\xi), K(\xi)$ denote the number of intervals and the constant in hypothesis $F$. Then $f$ is said to satisfy hypothesis $U F$ if $n(\xi)$ and $K(\xi)$ are uniformly bounded for $\xi \in X$; i.e. there exist constants $n$ and $K$ such that

$$
n(\xi) \leqq n, K(\xi) \leqq K, \xi \in X
$$

We refer to Rynne [6, Lemma 2.3] for relatively simple conditions under which hypothesis UF is satisfied.

\section{The fundamental estimate and results}

Suppose we have an index $i=(i, j)$ for which $\lambda^{i}=\left(\lambda^{i}, \mu^{i}\right) \in D_{\delta}$. With $t^{i}=\mu^{i} / \lambda^{i}$ we obtain from (5)

$$
k_{1}^{2}\left(t^{i}\right) \lambda^{i}\left(t^{i}\right)=i^{2} \pi^{2}-k_{1}^{2}\left(t^{i}\right)\left[q_{1} / s_{1}\right]+k_{1}^{2}\left(t^{j}\right)\left[\frac{\left(s_{1}^{1 / 4}\right)^{\cdot \cdot}}{s_{1}^{1 / 4}}\right]+a_{i}^{1}\left(t^{i}\right),
$$

and with the help of (7) and (9) we come to a cubic equation for $\psi_{1}=\phi_{1}\left(\lambda^{i}, \mu^{i}\right) /\left(\lambda^{i}\right)^{1 / 2}$, viz.

$$
\alpha_{1}\left(t^{i}\right) \psi_{1}^{3}-\lambda^{i} \psi_{1}^{2}-\beta_{i}\left(t^{i}\right) \psi_{1}+\gamma_{1}\left(t^{i}\right)=0
$$

where $\alpha_{1}, \beta_{1}$ are continuous and hence bounded functions of $t \in\left[t_{0}+\delta, t_{1}-\delta\right]$ and $\gamma_{1}(t)=i^{2} \pi^{2}+a_{i}^{1}(t)$. Note that it is an easy consequence of [5, Theorem $1, \mathrm{p}$. 51] that $a_{i}^{1}(t)$ is uniformly bounded in both $i$ and $t$. The upshot is that Cardano's formula can be applied to (11) and, after some cumbersome calculation, yields a solution

$$
\psi_{1}=\left(\gamma_{1} / \lambda^{i}\right)^{1 / 2}+O\left(1 / \lambda^{i}\right)
$$

or alternatively, 


$$
\begin{aligned}
\phi_{1}\left(\lambda^{i}\right)= & \sqrt{\gamma_{1}}+O\left(1 / \sqrt{\lambda^{i}}\right) \\
= & i \pi+O(1 / i)+O\left(1 / \sqrt{\lambda^{i}}\right) .
\end{aligned}
$$

Classical estimates for the eigenvalues of the problem (4) yield $\lambda^{i}=O\left(i^{2}\right)$, and so we obtain

$$
\phi_{1}\left(\lambda^{i}\right)=i \pi+O(1 / i)
$$

A similar analysis commencing with (6) yields

$$
\phi_{2}\left(\lambda^{i}\right)=j \pi+O(1 / j)
$$

so that in combination we have

$$
\phi\left(\lambda^{\mathbf{i}}\right)=\mathbf{i} \pi+O(1 /\|\mathbf{i}\|)
$$

or

$$
\begin{gathered}
i=\pi^{-1} \phi\left(\lambda^{i}\right)+O(1 /\|\mathbf{i}\|), \\
\phi\left(\lambda^{i} /\|\mathbf{i}\|^{2}\right)=\pi \frac{i}{\|i\|}+O\left(\|\mathbf{i}\|^{-2}\right) .
\end{gathered}
$$

Equations (12) and (13) correspond to Rynne's estimates (3.4), (3.5) where the order terms were $O(1)$ and $O\left(\|\mathbf{i}\|^{-1}\right)$ respectively. Thus (12) and (13) contain the improvement of Rynne's estimates we were seeking for eigenvalues in $D_{\delta}$. In some sense (12) and (13) are also best possible for in the special case $A_{1}=1, B_{1}=0, A_{2}=0, B_{2}=1$, the system is "diagonal" (and, in essence, unlinked), and equation (12) then yields the standard results for Sturm-Liouville problems

$$
\sqrt{\lambda_{i}}=\pi i+O(1 / i)
$$

The concluding arguments now follow those of Rynne $[6, \S 3]$ and we can omit details being content with statements of results. Note that if we assume the functions

$$
(x, \lambda) \rightarrow \lambda A_{1}(x)-\mu B_{1}(x), \quad(x, \lambda) \rightarrow-\lambda A_{2}(x)+\mu B_{2}(x)
$$

defined on $U \times \bar{Q}$ satisfy hypothesis UF, then $\phi: \bar{Q} \rightarrow \overline{\mathbf{R}}_{+}^{2}$ is a homeomorphism ([5, Theorem 3.1]). We can now conclude with our main results.

Theorem 3.1. (cf. [6, Theorem 3.1]). Suppose that the functions (14) satisfy hypothesis $U F$. Then the eigenvalues of the multiparameter problem (1), (2) satisfy

$$
\lambda^{i}=\pi^{2} \phi^{-1}(\mathbf{i})+O\left(\|\mathbf{i}\|^{2 / 3}\right)=\pi^{2}\|\mathbf{i}\|^{2} \phi^{-1}(\mathbf{i} /\|\mathbf{i}\|)+O\left(\|\mathbf{i}\|^{2 / 3}\right)
$$


for all $\mathbf{i} \neq 0$ such that $\lambda^{i} \in D_{\delta}$. If $\varepsilon>0$ then for all $\mathbf{i}=(i, j)$ with $i \geqq \varepsilon, j \geqq \varepsilon$ for which $\lambda^{i} \in D_{\delta}$,

$$
\lambda^{i}=\pi^{2} \phi^{-1}(\mathbf{i})+\varepsilon^{-1} O(1)=\pi^{2}\|\mathbf{i}\|^{2} \phi^{-1}(\mathbf{i} /\|\mathbf{i}\|)+\varepsilon^{-1} O(1) .
$$

Suppose that for all $\lambda \in \bar{Q}$ with $\|\lambda\| \leqq 1$ and for all $\gamma \in[0,1]$ the sets $\left\{x \in U \mid \lambda A_{1}(x)-\right.$ $\left.\mu B_{1}(x) \geqq-\gamma\right\},\left\{x \in U \mid-\lambda A_{2}(x)+\mu B_{2}(x) \geqq-\gamma\right\}$ have Lebesgue measure at least $c \gamma^{1 / 2}$ for some constant c. Then for all $\mathbf{i} \neq 0$ with $\lambda^{i} \in D_{\delta}$,

$$
\lambda^{i}=\pi^{2} \phi^{-1}(\mathbf{i})+O(1)=\pi^{2}\|\mathbf{i}\|^{2} \phi^{-1}(\mathbf{i} /\|\mathbf{i}\|)+O(1) .
$$

Theorem 3.2 (cf. [6, Theorem 3.2]). If $A_{1}, B_{1}, A_{2}, B_{2}$ are analytic, then the results of Theorem 3.1 hold.

\section{Concluding remarks}

It is natural to ask to what extent do the asymptotic forms of the eigenvalues given in Theorem 3.1 characterise all possible Dirichlet spectra? This question has important implications for the following two parameter inverse spectral problem:

Given coefficients $A_{i}, B_{i}, i=1,2$, to what extent is a point $\left\{q_{1}, q_{2}\right\} \in L^{2} \times L^{2}$ determined by its two parameter Dirichlet spectrum?

This problem is a natural generalisation of the 1-parameter "isospectral mapping" problem described and completely solved in [5]. We hope to develop this generalisation in subsequent papers.

\section{REFERENCES}

1. P. A. Binding and P. J. Browne, Positivity results for determinantal operators, Proc. Roy. Soc. Edinburgh 81 A (1978), 267-271.

2. P. A. Binding and P. J. Browne, Comparison cones for multiparameter eigenvalue problems, J. Math. Anal. Appl. 77 (1980), 132-149.

3. M. Faierman, On the distribution of the eigenvalues of a two-parameter system of ordinary differential equations of the second order, SIAM J. Math. Anal. 8 (1977), 854-870.

4. M. Faierman, Distribution of eigenvalues of a two-parameter system of differential equations, Trans. Amer. Math. Soc. 247 (1979), 45-86.

5. J. Poschel and E. TRubowirz, Inverse Spectral Theory (Academic Press, London, 1987).

6. B. P. Rynne, The asymptotic distribution of the eigenvalues of right definite multiparameter Sturm-Liouville systems, Proc. Edinburgh Math. Soc., to appear.

Department of Mathematics and Statistics UNIVERSITY OF CALGARY

Calgary Alberta

Canada T2N 1N4
Department of Mathematics and Computer Science

UNIVERSITY OF DUNDEE

DUNDEE DD1 4HN

Scotland 